\title{
PROGRAMA DE REGIONALIZAÇÃO NA ASSISTÊNCIA NEONATAL UMA VISÃO DO SERVIÇO SOCIAL NA ATENÇÃO À SAÚDE
}

\author{
Elaine Aparecida de Carvalho Salcedo ${ }^{1}$ \\ Sergio Tadeu Martins Marba ${ }^{2}$
}

\begin{abstract}
RESUMO
O artigo contextualiza a trajetória de um programa de Neonatologia do Centro de Atenção Integral à Saúde da Mulher, norteado por políticas públicas visando a humanização e qualidade dos serviços prestados, como também a ação do assistente social como agente educador e executor deste processo. Esse programa é viabilizado entre Serviços de Saúde que através de um sistema de Regionalização do atendimento Neonatal pela referência e contra-referência atuam integrados para realizar a transferência de recém-nascidos internados conforme necessidades e competências. Esta discussão poderá enfatizar o trabalho multiprofissional e a necessidade de um planejamento regional entre cidades e Serviços de Saúde para execução dessa proposta.
\end{abstract}

PALAVRAS-CHAVE: Organização Regional; Trabalho Multiprofissional; Neonatologia.

\section{INTRODUÇÃO}

O Centro de Atenção Integral à Saúde de Mulher (CAISM) é um Hospital de nível terciário da Universidade Estadual de Campinas - UNICAMP, ligado a Faculdade de Ciências Médicas através dos diversos Departamentos tais como: Tocoginecologia e Pediatria.

A Área de Neonatologia é uma extensão deste último e conta com uma estrutura que compreende uma unidade de internação de alto e médio risco com capacidade de 30

\footnotetext{
${ }^{1}$ Assistente social da Neonatologia do Centro de Atenção Integral à Saúde da Mulher.

${ }^{2}$ Diretor da Neonatologia do Centro de Atenção Integral à Saúde da Mulher e Professor Assistente Doutor do Departamento de Pediatria da Faculdade de Ciências Médicas/ UNICAMP.

\begin{tabular}{llllll}
\hline Serviço Social \& Saúde & Campinas & v. 4 & n. 4 & p. 1-156 & Maio 2005
\end{tabular}
} 
leitos, alojamento conjunto com 24 leitos para recém-nascidos de baixo risco, atendimento em sala de parto, além de ambulatórios especializados para atender recém-nascidos prematuros ou com outras patologias que necessitem de continuidade na assistência neonatal pós-alta hospitalar.

Enfocando esta Área o presente artigo tem como proposta apresentar o Programa de Regionalização da Assistência Neonatal desde a sua implantação em janeiro de 1996 até outubro de 2004, analisando os dados e resultados desse trabalho de parceria com outras Unidades de Saúde integradas em um sistema de referência e contra-referência para atender demandas desta especialidade médica.

A origem deste programa nos reporta ao ano de 1988 quando foi realizada uma pesquisa no CAISM e junto aos Serviços de Saúde que prestavam assistência maternoinfantil em um raio de $150 \mathrm{Km}$ da UNICAMP, onde foram levantados os dados estatísticos de partos e de nascimentos com incidência de baixo peso, bem como o número de óbitos neonatais neste período (FACCHINI, 1988 ).

Neste estudo também foi verificado que a cidade de Campinas apresentava condições para uma assistência hierarquizada e regionalizada nessa área, por contar com uma maior concentração de serviços de saúde materno-infantil com nível terciário, além de ter várias rodovias na região que facilitariam os deslocamentos de usuários.

Outro recurso favorável disponibilizado pelo CAISM desde 1986 para implantar essa estrutura foi o Centro de Repouso, hoje denominada Seção de Apoio Social (SAS), local que possibilita estadia sem custos às pacientes em acompanhamento nas diversas áreas desse Hospital que necessitam desse recurso para complementaridade da assistência, incluindo entre estas as gestantes e puérperas.

Estes dados subsidiaram o planejamento de ações pelo CAISM visando a implantação de um Programa de Regionalização na Assistência Neonatal envolvendo Serviços de Saúde de Campinas e cidades circunvizinhas.

Esse Centro com foco neste objetivo realizou a partir de 1990 crescentes investimentos em reformas para adequação da área física, na aquisição de equipamentos, contratação e treinamento de recursos humanos, visando ampliar a capacidade de 
atendimento da unidade de internação e ambulatórios, assim como melhorar a resolutividade da assistência para atender esta demanda.

Concomitantemente foram realizadas visitas pela direção da Neonatologia aos Hospitais da região para levantamento da disponibilidade de leitos, dos serviços prestados e da demanda, executando assim articulações em rede para sensibilização e parcerias com vistas à concretização desta proposta.

Essas ações gradativamente consolidaram em 1996 a implantação do Programa de Regionalização da Assistência Neonatal, ou seja, a organização do sistema de referência pelo CAISM que daria prioridade aos atendimentos de maior complexidade nas especialidades médicas de competência e contra-referência assumida pelos hospitais da região com estrutura para atender essa demanda, conforme tecnologias e recursos disponíveis viabilizando assim a adequação dos atendimentos das gestantes e puérperas.

Nesta proposta os Hospitais realizam as transferências de gestantes e recémnascidos que necessitam de cuidados de terapia intensiva, após discussão do caso com médico responsável pelo plantão em conformidade a esse sistema de atenção à saúde, cabendo ao CAISM a assistência terciária e no momento em que estes se encontram em cuidados de semi-intensivo são transferidos para Hospitais de atenção primária ou secundária, situados na cidade de residência ou referência dos usuários.

Os objetivos do Programa são:

- Otimizar a utilização e disponibilidade dos leitos na Unidade de Terapia Intensiva Neonatal do CAISM com a integração das ações da equipe multiprofissional, viabilizando a transferência de recém-nascidos em sistema de referência e contrareferência;

- Prestar assistência com qualidade e humanização durante período de seguimento na unidade, realizando ações e parcerias com Serviços de Saúde da região conforme avaliação sócio-econômica dos usuários, garantindo a continuidade deste atendimento a nível ambulatorial conforme necessidade do recém-nascido e estrutura disponível nos serviços referenciados;

- Redirecionar a demanda dos recém-nascidos em cuidados intermediários que possibilita maior resolutibilidade na assistência e diminuição na incidência de infecção hospitalar. 
Na operacionalização deste Programa para subsidiar as condutas clínicas da equipe médica foram definidos os seguintes critérios para solicitação de transferência de recém-nascidos internados na Neonatologia:

- Peso acima de 1500 gramas, sem necessidade de monitorização contínua;

- Estar sob cuidados intermediários para ganho ponderal, recebendo apenas alimentação enteral;

- Estar com respiração espontânea em ar ambiente ou em baixas concentrações de oxigênio;

- Ter o consentimento dos pais e vaga autorizada no serviço de contra-referência. (FACCHINI,1988)

Durante o período compreendido entre a internação e seguimento ambulatorial do recém-nascido na Unidade os pais e familiares são atendidos por uma equipe multiprofissional composta por profissionais das áreas de: Medicina, Enfermagem, Serviço Social, Psicologia, Fisioterapia, Fonoaudiologia e Nutrição.

Seguindo essa proposta esses profissionais integram as abordagens na assistência para promover a ação educativa e o apoio no período de seguimento na unidade. Outro foco é a ação terapêutica concretizada nos grupos com os pais e familiares de bebês internados realizados sistematicamente duas vezes por semana. Essa atividade possibilita a troca de experiências entre equipe e usuários, além de propiciar espaços de escuta e suporte emocional ao compartilhar sentimentos e vivências.

O assistente social na sistematização de rotinas realiza entrevistas e contatos com os responsáveis para: acolhimento e estabelecimento de vínculo, como também avaliação sócio-econômica e orientações que somadas as outras abordagens permitem intervir de acordo com situações emergentes e necessidades relatadas.

Desta forma o assistente social subsidia também a definição de condutas ao interpretar a realidade vivenciada pelos familiares nas discussões de casos com a equipe, além de atuar fazendo a correlação com as Políticas Sociais para buscar alternativas para efetivar o tratamento proposto.

Outra ação executada é a articulação constante com rede de serviços afins da cidade de origem dos usuários para levantamento de recursos existentes, como também

\begin{tabular}{llllll}
\hline Serviço Social \& Saúde & Campinas & v. 4 & n. 4 & p. 1-156 & Maio 2005 \\
\hline
\end{tabular}


para viabilizar parcerias no atendimento e garantir continuidade na assistência aos recémnascidos e familiares. Esses procedimentos são realizados através de contatos telefônicos, encaminhamento de relatórios dos profissionais, documentos e prescrições médicas.

Neste entrosamento o assistente social busca complementar as abordagens realizadas na Unidade que associadas a execução de outras intervenções, podendo citar, a visita domiciliar possibilitam convocação, avaliação das condições habitacionais, da dinâmica familiar e situação sócio-econômica para inserção em programas sociais, bem como a concessão de transportes e outros recursos, citados a seguir: medicação, cesta básica, fórmula láctea, aspirador, sonda e outros equipamentos necessários para efetivação da transferência ou alta.

Enfatizando o Programa de Regionalização realizado pelo CAISM em parceria com outros Serviços de Saúde da região a seguir descreveremos as ações para execução da transferência de recém-nascidos.

Conforme evolução clínica destes os responsáveis são orientados sobre os critérios, trâmites e objetivos do Programa de Regionalização, sendo avaliado ainda pelo Serviço Social as condições favoráveis ou fatores interferentes para viabilização da transferência.

O médico com base nos critérios do Programa indica o momento para realização deste procedimento e executa o preenchimento de impresso contendo as informações relativas ao recém-nascido, tratamento em vigência e os equipamentos necessários no seguimento.

Considerando que estes se encontram em condições estáveis e em cuidados intermediários o assistente social executa contatos telefônicos com pediatra responsável pela concessão de vaga no hospital de contra-referência para discussão do caso, solicitação de transferência e esclarecimentos das rotinas do CAISM nesse processo. No caso do hospital requerer complementação de dados com o médico será realizado contato telefônico pelo profissional citado.

Algumas cidades utilizam a Central de Vaga para administrar esse procedimento que é acionado inicialmente através de telefone para discussão do caso e em seqüência com o encaminhamento de fax contendo documento específico preenchido pelo médico \begin{tabular}{llllll}
\hline Serviço Social \& Saúde & Campinas & v. 4 & n. 4 & p. 1-156 & Maio 2005
\end{tabular} 
responsável para ser articulado com o Hospital de destino para a concessão de vaga ao recém-nascido.

Na ocasião da transferência se os responsáveis pelo recém-nascido não se encontrarem na unidade é executada a convocação destes para atendimento pelo Serviço Social para esclarecimentos relativos ao Programa e a realização de procedimentos pela equipe.

Outra rotina da unidade é a elaboração de relatório médico que é fornecido aos responsáveis pelo recém-nascido na alta como também encaminhado ao Hospital na situação de transferência, assim como são garantidos os agendamentos para atendimentos ambulatoriais em especialidades médicas ou procedimentos no caso destes serviços inexistirem na cidade de origem dos usuários.

Para finalizar a transferência a enfermagem executa a formalização do consentimento, as orientações quanto os cuidados com o recém-nascido, a organização do acompanhamento médico ou de enfermeira durante o percurso, como também a disponibilização de transporte do CAISM.

Esse Programa em vigência efetiva a proposta contida na Norma Operacional á Saúde do Sistema Único de Saúde que atualizou a regulamentação da assistência e busca ampliar a responsabilidade dos municípios, além de garantir o acesso aos serviços de atenção á saúde e estabelecer a regionalização e organização funcional do sistema (NOAS, 2001).

\section{METODOLOGIA}

O trabalho apresentado tem como base a análise de projetos, pesquisas, censo da área e também a documentação utilizada como instrumental técnico do Serviço Social da Neonatologia, composta pelo documento perfil sócio-econômico, relatórios e estatística dos atendimentos.

A metodologia para execução dessa pesquisa considerou o universo de 661 casos correspondentes as transferências executadas no Programa citado no período de janeiro de 1996 até outubro de 2004 restrita nesse momento ao levantamento de dados dos relatórios estatísticos diários do Serviço Social e listagem dos usuários internados na Neonatologia.

\begin{tabular}{llllll}
\hline Serviço Social \& Saúde & Campinas & v. 4 & n. 4 & p. 1-156 & Maio 2005 \\
\hline
\end{tabular}


O censo utilizado nessa pesquisa contém registros de dados dos recém-nascidos e dos procedimentos referidos, sendo: nome; número de registro do prontuário; datas do parto e transferência; diagnóstico na internação; peso de nascimento e ganho ponderal atualizado; cidade de origem; hospital e cidade de destino na transferência.

O levantamento dos dados delimitados possibilitou determinar o universo das transferências executadas, a área de abrangência e a distribuição percentual da taxa de ocupação antes e após a implantação do Programa.

\section{RESULTADOS}

Na seqüência apresentaremos os resultados dessa pesquisa através de representação gráfica, comentários e considerações finais buscando subsidiar Políticas de Saúde, a hierarquização e regionalização proposta pelo SUS, como também estudos posteriores nesse universo temático.

Figura 1 - Número de transferências de recém-nascidos para unidades secundárias segundo mês e ano.

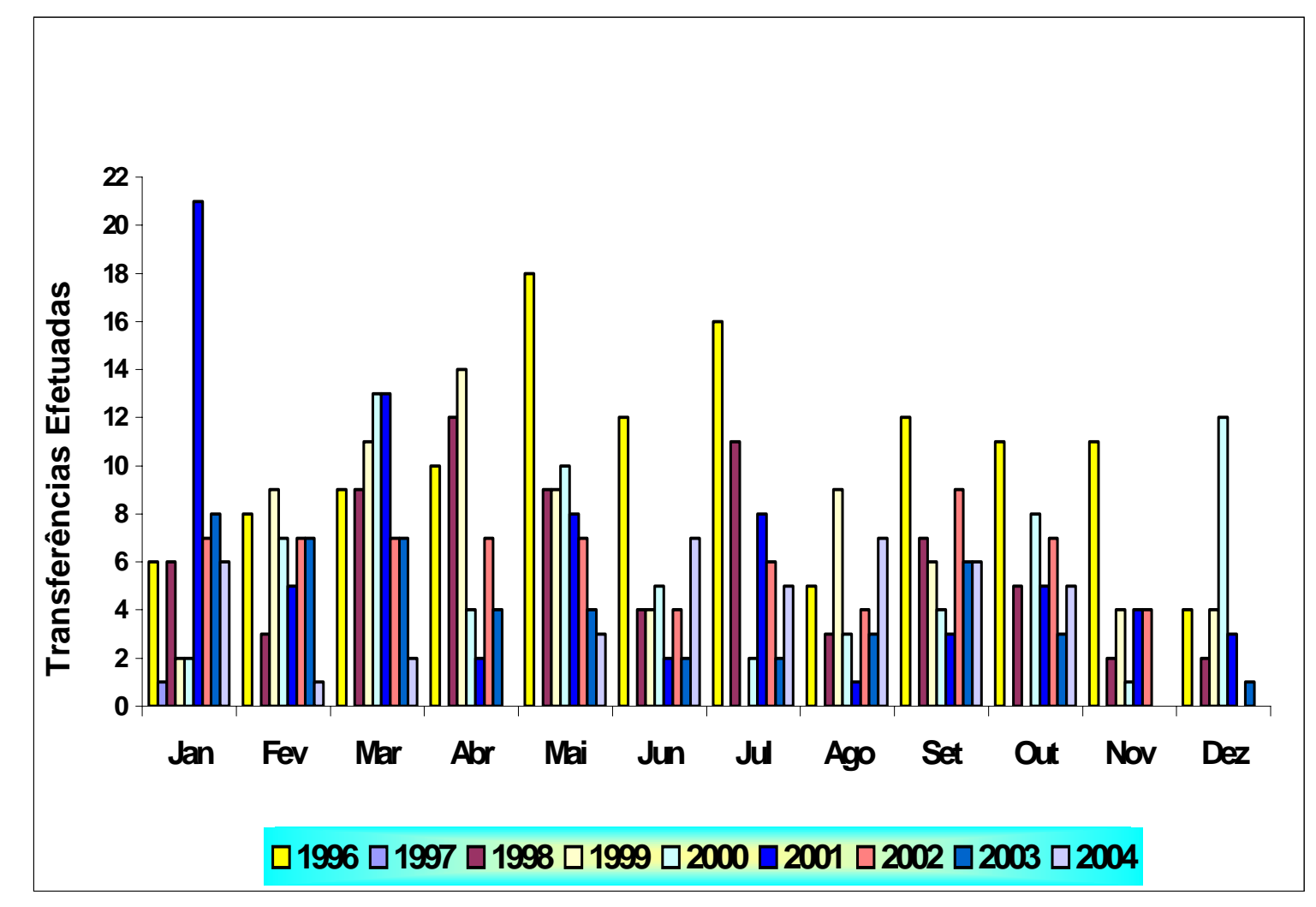

Serviço Social \& Saúde Campinas

$\begin{array}{lll}\text { v. } 4 & \text { n. } 4 & \text { p. } 1-156\end{array}$

Maio 2005 
No período analisado foram realizadas um total de 661 transferências (Figura 1). Esses dados possibilitaram a constatação da efetividade do Programa de Regionalização considerando que os recém-nascidos encaminhados aos Serviços de Saúde da região estavam em condições clínicas estáveis em cuidados de semi-intensivo, conforme critérios estabelecidos e que as vagas foram ocupadas por outros com patologias que necessitam de equipamentos e tecnologia disponíveis na Unidade de Terapia Intensiva à nível terciário pela complexidade.

Tabela 2 -Distribuição percentual das transferências segundo cidades de contra-referência.

\begin{tabular}{|l|c|c|}
\hline \multicolumn{1}{|c|}{ Cidades de Origem } & Transferências & $\%$ \\
\hline Sumaré & 124 & $18,76 \%$ \\
\hline Paulínia & 111 & $16,79 \%$ \\
\hline Campinas & 87 & $13,16 \%$ \\
\hline Hortolândia & 28 & $4,24 \%$ \\
\hline Pedreira & 22 & $3,33 \%$ \\
\hline Itapira & 20 & $3,03 \%$ \\
\hline Santa Bárbara do Oeste & 17 & $2,57 \%$ \\
\hline Cosmópolis & 16 & $2,42 \%$ \\
\hline Indaiatuba & 15 & $2,27 \%$ \\
\hline Vinhedo & 14 & $2,12 \%$ \\
\hline Amparo & 11 & $1,66 \%$ \\
\hline Itatiba & 10 & $1,51 \%$ \\
\hline Jaguariúna & 9 & $1,36 \%$ \\
\hline Jundiaí & 9 & $1,36 \%$ \\
\hline Santo Antonio da Posse & 8 & $1,21 \%$ \\
\hline Rio Claro & 8 & $1,21 \%$ \\
\hline Águas de Lindóia & 8 & $1,21 \%$ \\
\hline Outras 3 cidades (7 por localidade) & 21 & $3,18 \%$ \\
\hline Outras 3 cidades (6 por localidade) & 18 & $2,72 \%$ \\
\hline Outras 2 cidades (5 por localidade) & 10 & $1,51 \%$ \\
\hline Outras 5 cidades (4 por localidade) & 20 & $3,03 \%$ \\
\hline Outras 7 cidades (3 por localidade) & 21 & $3,18 \%$ \\
\hline Outras 14 cidades (2 por localidade) & 28 & $4,24 \%$ \\
\hline Outras 26 cidades (1 por localidade) & 26 & $3,93 \%$ \\
\hline Total & 661 & $100,00 \%$ \\
\hline
\end{tabular}


No período analisado foi constatado que Sumaré (Tabela 2) foi a cidade com a maior prevalência de transferências realizadas totalizando 18,76 \% destes procedimentos.

Considerando essa tabulação e somando a incidência de transferências efetuadas às cidades circunvizinhas deste município foi confirmado a necessidade de implantação de outro Hospital de referência nessa região, fato ocorrido em 2001 com a inauguração do Hospital Estadual de Sumaré, que em parceria com a UNICAMP através de convênio passou a atender demandas desta área.

A abrangência deste atendimento incluindo cidades de outro estado de maior proximidade (Minas Gerais) demonstra que a sistematização proposta pelo Programa de Regionalização possibilita garantir o acesso aos usuários às ações e serviços necessários para efetivar a resolução dos seus problemas de saúde ao integrar cuidados nos diversos níveis de atenção, otimizando os recursos disponíveis o que amplia a conformidade e articulação entre Unidades envolvidas.

Figura 3 - Número de transferências de recém-nascidos para unidades secundárias segundo ano.

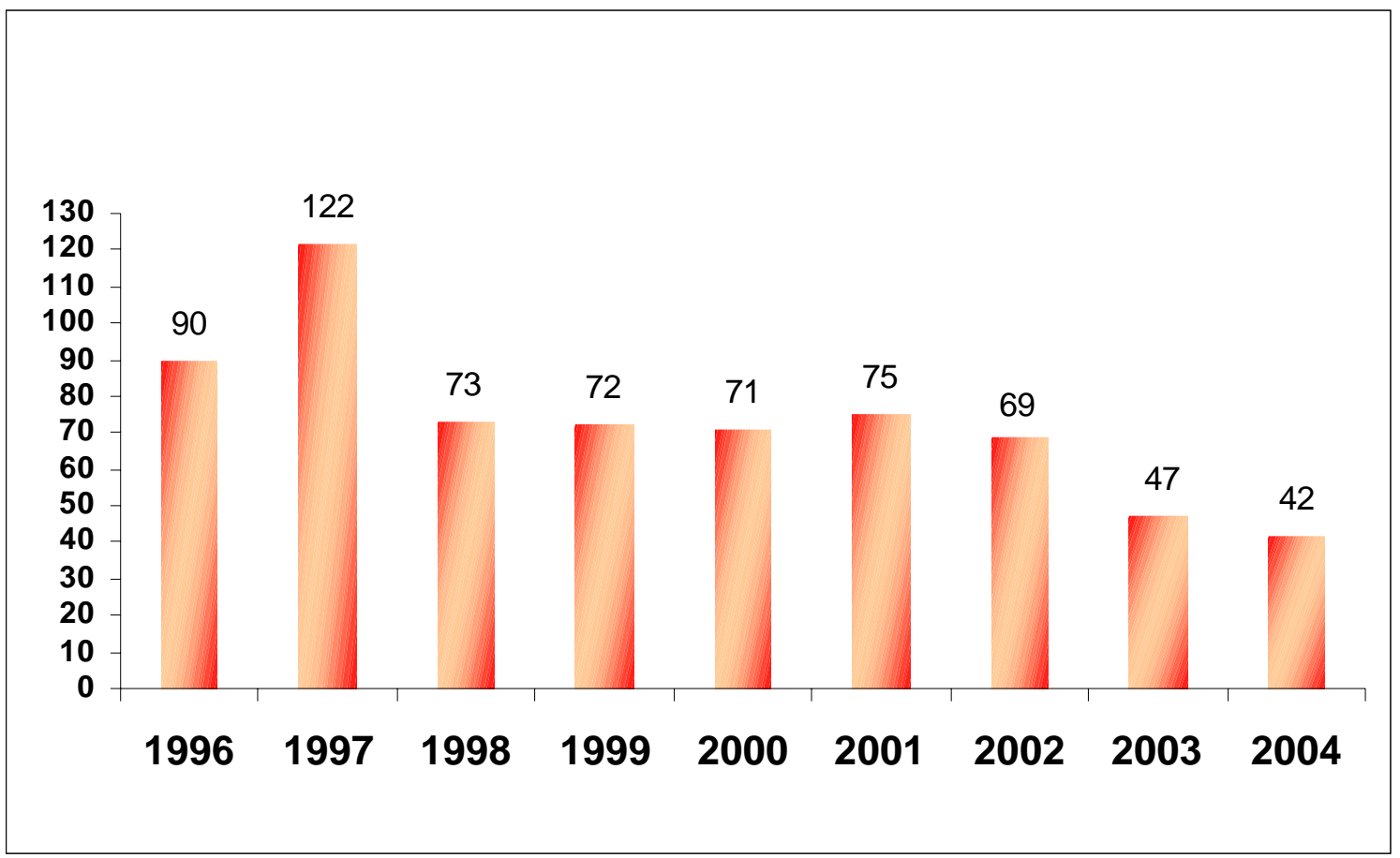

Serviço Social \& Saúde Campinas v. 4 n. 4 p. $1-156$ Maio 2005 
A partir de 2001 foi verificado diminuição no número de internações de recémnascidos na Unidade e conseqüentemente nas transferências realizadas aos Hospitais de nível primário e secundário (Figura 3).

Com isso podemos observar que a taxa de ocupação atual da unidade de internação da Neonatologia do CAISM em comparação ao período de implantação do Programa de Regionalização se mantém em níveis aceitáveis permitindo atender recém-nascidos encaminhados pela referência com condições adequadas á nível de recursos humanos, equipamentos e serviços disponíveis (Figura 4).

Figura 4 - Distribuição percentual da taxa de ocupação em três épocas distintas antes e após implantação do Programa de Regionalização.

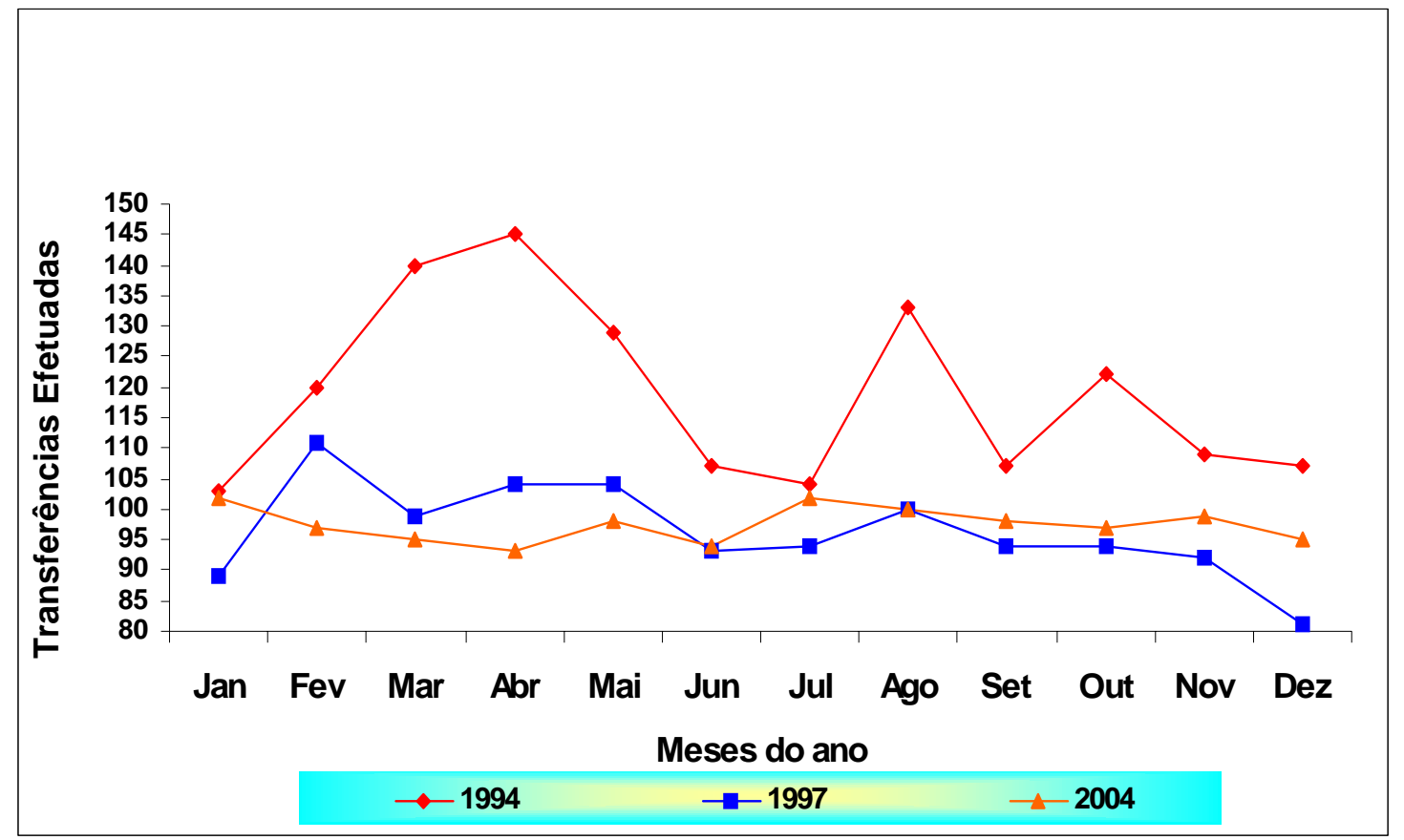

Para complementação de dados vale ressaltar que o CAISM conforme diretrizes institucionais e com base nos resultados do Programa de Regionalização da Neonatologia tem de forma crescente viabilizado essa sistematização na Obstetrícia através da articulação com Serviços de Saúde, para garantir assistência às gestantes com necessidade de pré-natal especializado pelas patologias de risco, o que incidiu significativamente na ampliação da 
área de abrangência e melhoria nas condições de partos e nascimentos pela atenção e cuidados prestados na gestação.

Outras variáveis consideradas a serem analisadas posteriormente somando a estes dados se relacionam à ampliação de Unidades de Terapia Intensiva e Semi-intensiva em Hospitais de atenção secundária e primária existentes na região, assim como a atuação do Hospital Estadual de Sumaré como Centro de referência assumindo demandas desta área em diversas especialidades médicas, incluindo a Neonatologia.

\section{CONSIDERAÇÕES FINAIS}

O Programa de Regionalização na Assistência Neonatal tem concretizado nesses anos os objetivos de garantir o acesso universal aos Serviços de Saúde e a integração de ações nos diversos níveis de atenção, atendendo as demandas conforme competências com uma maior abrangência.

Esta organização possibilita ainda o processo de descentralização proposto pelo Sistema Único de Saúde que na regionalização e hierarquização da rede de serviços redefine responsabilidades e recursos nos âmbitos: municipal, estadual e federal para efetivar a assistência à saúde.

Outro fator relevante é que ao garantir a assistência neonatal e a sua continuidade através da articulação com Unidades de Saúde a humanização e a qualidade de vida também estão sendo valorizadas.

Essas ações tem possibilitado a otimização da utilização de leitos na unidade e também na assistência, pela adequação da demanda aos equipamentos e recursos humanos disponíveis, assim como a diminuição na taxa de infecção hospitalar.

É importante ressaltar que na transferência de recém-nascidos aos serviços de contra-referência existe redução da distância percorrida pelos usuários no deslocamento ao hospital e também dos custos com o transporte, principalmente aos residentes em outras cidades, contribuindo para uma maior constância nas visitas e contato com o recémnascido, bem como a integração com a equipe e incentivo ao aleitamento buscando melhorar a qualidade de vida e concretizar a proposta de atenção à integral à saúde.

\begin{tabular}{llllll}
\hline Serviço Social \& Saúde & Campinas & v. 4 & n. 4 & p. 1-156 & Maio 2005
\end{tabular} 
O Serviço Social inserido na equipe multiprofissional ao interpretar os aspectos sociais e realizar a articulação com instituições e Serviços de Saúde para parcerias tem legitimado suas ações colaborando na sistematização de rotinas e efetivação de uma assistência mais humanizada.

\begin{abstract}
The article contextualize the trajectory of Neonatology care programs the Centro de Atenção Integral à Saúde da Mulher - CAISM in Campinas, Brazil and the treatment guided by public politics, aimed at humanizing and the quality of services provided, also the action the social worker as an educator and executor in this process. This program is viable between Health Service's help with a system of regional division on attention to neonatal for reference and counter-reference working integrated to make possible transference of news born in a Hospital conform with necessities and competencies. This discussion should emphasize the multi-professional work and the necessity for regional planning between cities and Health Service's for this proposal.
\end{abstract}

KEYWORDS: Regional Organization; Multi- professional Work; Neonatology.

\title{
REFERÊNCIAS BIBLIOGRÁFICAS
}

FACCHINI, F. P..Manual de Neonatologia UNICAMP CAISM In: MARBA, S.T. M. e FILHO, F. M. (Orgs.) Regionalização da Atenção Neonatal. São Paulo: Livraria e Editora Revinter LTDA, 1998. p.3-6

SWYER, P. R. The Regional Organization of Special care for the neonate. In: The Pediatric Clinics of North America. Toronto, Saunders Company, 1970, v. 7.

ALMEIDA, M F B; REGO, M.A; COSTA H P.F . Documento Científico do Departamento de Neonatologia da Sociedade Brasileira de Pediatria Infra-estrutura para Atendimento Integral ao recém-nascido; novembro de 2004.

MARBA, S. T. .M. et al. Resultados de um Programa de Regionalização da Assistência Neonatal em UTI de nível terciário. In Anais XVI CONGRESSO BRASILEIRO DE PERINATOLOGIA, XIII REUNIÃO DE ENFERMAGEM PERINATAL, 1998, Salvador.

\begin{tabular}{llllll}
\hline Serviço Social \& Saúde & Campinas & v. 4 & n. 4 & p. 1-156 & Maio 2005 \\
\hline
\end{tabular}


BRASIL. Portaria SUS n ${ }^{0}$ 95, 26 de janeiro de 2001. Atualiza a regulamentação da assistência à saúde. Norma Operacional da Assistência à Saúde/ NOAS- SUS 01/2001. Brasília, 01 de 2001 p.4-5. Disponível em: < http: // www.saúde.pa.gov.br/ SUS/ Legisla\% C3\% A\% A30/ NOAS01_PT95.htm . Acesso em: 19. ago. 2004 
